\title{
Successful Combined Therapy of Cyclophosphamide and Cyclosporine for Acute Exacerbated Interstitial Pneumonia Associated with Dermatomyositis
}

\author{
Fumiko Tanaka, Tomoki Origuchi*, Kiyoshi Migita, Masahiro Tominaga, Atsushi Kawakami, \\ Yojiro KaWABE and Katsumi EGUCHI
}

\begin{abstract}
Interstitial pneumonia of polymyositis and dermatomyositis (PM/DM), especially the rapid progressive type, is a serious complication. We report a case of acute exacerbated interstitial pneumonia associated with DM. The respiratory distress condition was refractory to steroid pulse therapy and cyclosporine, however, there was a good response to additional intravenous cyclophosphamide pulse therapy (IVCY). We propose the possibility that the combination of these immunosuppressants might be useful for interstitial pneumonia with DM which is resistant to conventional steroid therapies.

(Internal Medicine 39: 428-430, 2000)
\end{abstract}

Key word: nonspecific interstitial pneumonia, KL-6

\section{Introduction}

Polymyositis and dermatomyositis (PM/DM) are systemic inflammatory diseases which involve mainly the skeletal system. Interstitial pneumonia frequently occurs in association with $\mathrm{PM} / \mathrm{DM}$. Interstitial pneumonia is a serious complication of $\mathrm{PM} / \mathrm{DM}$ and carries an especially poor prognosis in the rapid progressive type (1).

The pulmonary involvement is often refractory to conventional therapies. Although immunosuppressive drugs such as azathioprine, cyclosporine and cyclophosphamide are used together with corticosteroids in patients resistant to corticosteroids $(2-5)$, the effectiveness is controversial $(6,7)$. There also have been some attempt to apply intravenous cyclophosphamide pulse therapy (IVCY) to cases with interstitial pneumonitis-associated PM/DM $(1,3)$. Here, we present a case of steroid-resistant interstitial pneumonia complicated with DM that could be successfully treated with a complication of IVCY pulse steroids and cyclosporine.

\section{Case Report}

A 44-year-old woman was admitted to our hospital for dyspnea. In 1993, she was diagnosed as having DM because of skin rash, muscle weakness and elevation of muscle enzymes. Histological findings of her muscle were compatible with DM. At that time, interstitial pneumonia was revealed on chest Xrays and computed tomography (CT). She was treated with oral steroid, followed with $12.5 \mathrm{mg}$ of prednisolone per day.

In May 1998, she had dry cough and dyspnea. She was emergently admitted to our hospital again for rapidly progressive interstitial pneumonia, which was pointed out on chest Xrays and $\mathrm{CT}$.

On admission, her body temperature was $35.7^{\circ} \mathrm{C}$, pulse $78 /$ $\mathrm{min}$, blood pressure $90-54 \mathrm{mmHg}$ and respiration rate $30 / \mathrm{min}$. Her face was flushed and moon shaped, however, typical skin lesions of dermatomyositis were not seen. A crepitant rale was audible in bilateral mid-to-lower lung field areas. Neurological examination revealed proxismal muscle weakness with tenderness.

Laboratory examination revealed the following values: a leucocyte count of $17,300 / \mathrm{mm}^{3}$, red cell count $479 \times 10^{4} / \mathrm{mm}^{3}$, hemoglobin $11.5 \mathrm{~g} / \mathrm{dl}$, platelet count $43.9 \times 10^{4} / \mathrm{mm}^{3}$, ALT 48 IU/l, AST $59 \mathrm{IU} / l$, LDH $600 \mathrm{IU} / l$, CPK $367 \mathrm{IU} / l$, aldolase 9.4 IU/l, ALP $169 \mathrm{IU} / l$, CRP 5.28 mg/dl, KL-6 866 U/ml (normal range; $<500 \mathrm{U} / \mathrm{ml}$ ).

Serological test revealed positive anti-nuclear antibody $(\times 160$, speckled and cytoplasmic). Antibody for Jo-1 was negative. Serum cytomegalovirus antigen was not detected. Analysis of blood gases showed the following levels: $\mathrm{pH}$ 7.464, $\mathrm{PCO}_{2}$ $40.3 \mathrm{mmHg}, \mathrm{PO}_{2} 56.1 \mathrm{mmHg}, \mathrm{HCO}_{3} 28.3 \mathrm{mmHg}, \mathrm{SaO}_{2} 92.4 \%$ and $\mathrm{Aa}-\mathrm{DO}_{2} 46 \mathrm{mmHg}$ on room air. Urinalysis was normal. No bacteria (including mycobacterium tuberculosis) was de-

From the First Department of Internal Medicine, Nagasaki University School of Medicine and *School of Allied Medical Sciences, Nagasaki University, Nagasaki

Received for publication June 17, 1999; Accepted for publication December 24, 1999

Reprint requests shoud be address to Dr. Katsumi Eguchi, the First Department of Internal Medicine, Nagasaki University School of Medicine, 1-7-1, Sakamoto, Nagasaki 852-8501 


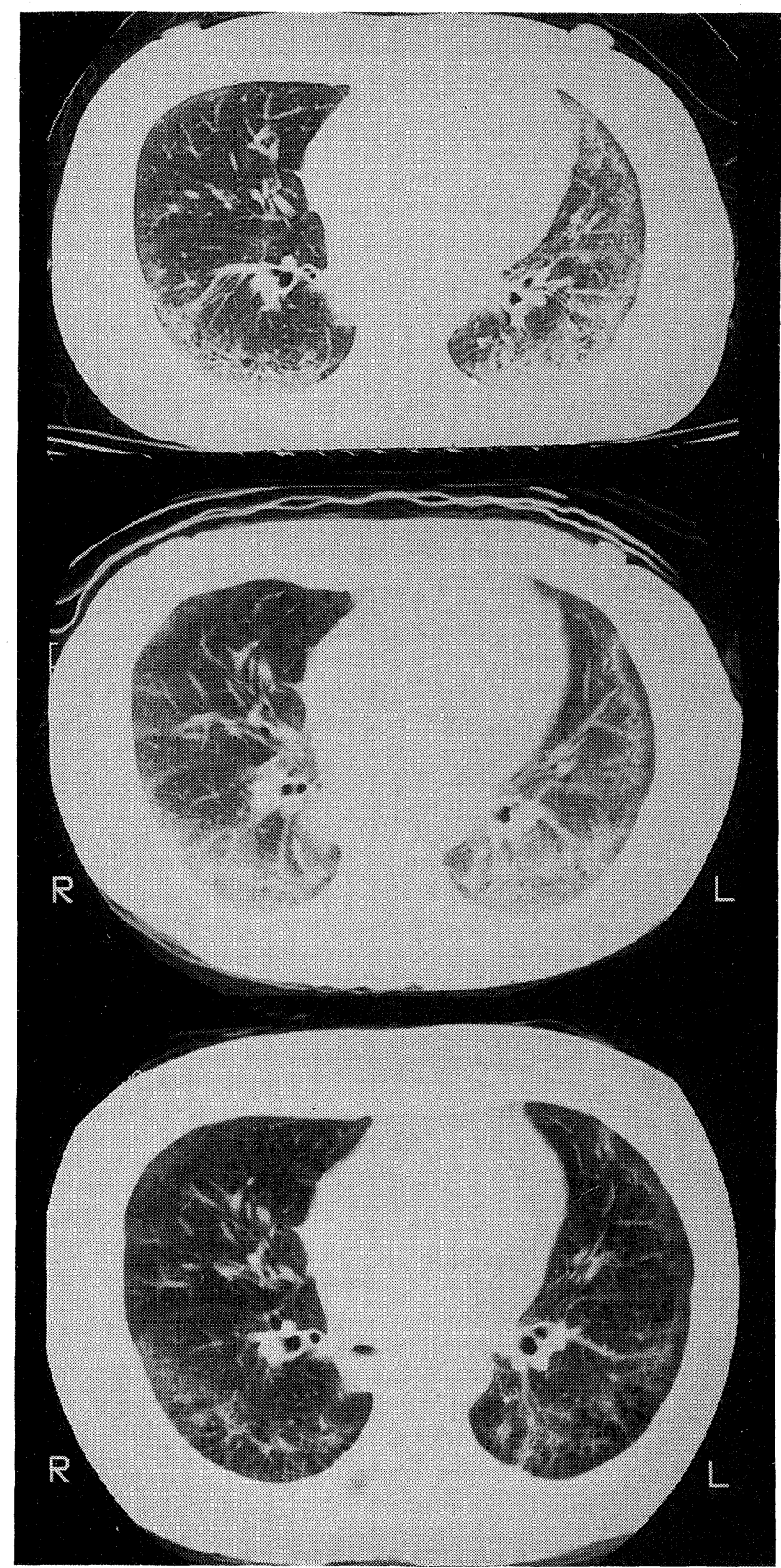

Figure 1. Chest C'T findings in May 1998 (on admission) (upper), in July 1998 (after treatment of steroid and cyclosporin A) (middle), and in August 1998 (one month after addition of intravenous cyclophosphamide pulse therapy (IVCY)) (lower) showing that interstitial pneumonia is rapidly progressive and improved after addition of IVCY.

tected in her sputum.

On a chest roentgenogram and a CT scan, reticular shadows were detected in the bilateral lower lung fields on admission, in May 1998 (Fig. 1, upper). Radiological studies revealed typi-

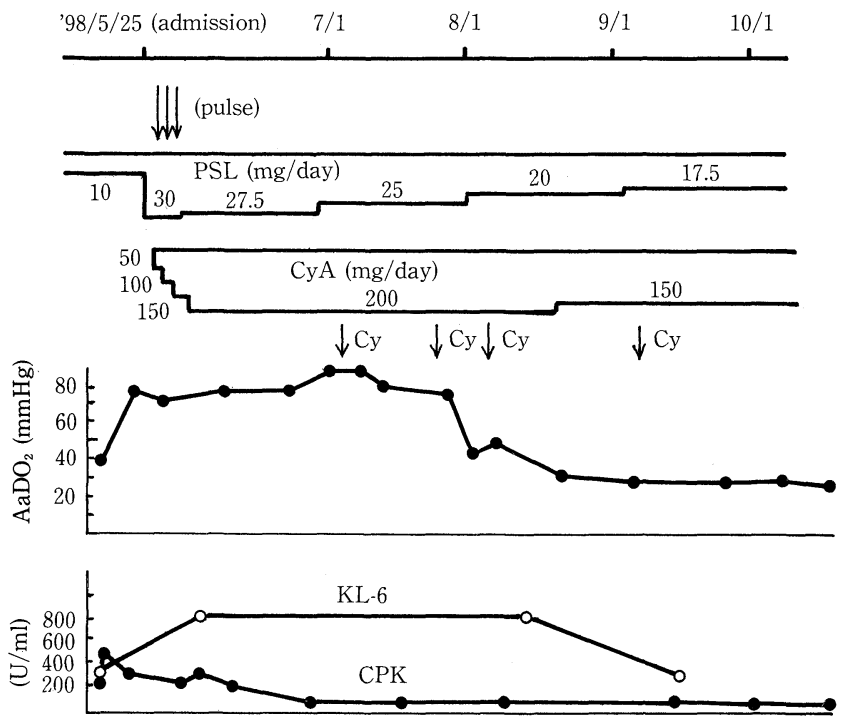

Figure 2. The clinical course of this case. Pulse: steroid pulse therapy, CyA: cyclosporin A, PSL: prednisolone, Cy: intravenous cyclophosphamide $(500 \mathrm{mg})$.

cal shadows of interstitial pneumonia. She was diagnosed as having DM complicated with interstitial pneumonia.

Although pulse therapy with methylprednisolone and oral prednisolone combined with cyclosporine was effective for DM, they proved to be ineffective for interstitial pneumonia (Fig. 2). Oxygen inhalation was required because of respiratory distress due to rapid progression of interstitial pneumonia (Fig. 1, middle). Therefore, after obtaining informed consent, IVCY (10 mg/kg i.v., 4 times) was performed, which proved to be successful. Respiratory failure due to interstitial pneumonia was gradually improved with normalization of $\mathrm{Aa}-\mathrm{DO}_{2}$ (Fig. 1, lower); she did not require further oxygen inhalation while prednisolone was tapered. Serum levels of KL-6 antigen, which is a novel indicator of the extent of clinical activity of interstitial pneumonia (8), were reduced in accordance with clinical disease activity. She was maintained on $17.5 \mathrm{mg}$ prednisolone and $150 \mathrm{mg}$ cyclosporine and discharged on October 1998. Therefore, stable pulmonary function and stable chest radiographic findings have been maintained for over 11 months by prednisolone (17.5 $\mathrm{mg}$ to $18 \mathrm{mg}$ ) and $150 \mathrm{mg}$ cyclosporine.

\section{Discussion}

Recently, cases of rapidly progressive interstitial pneumonia associated with PM/DM have been reported. Although they do not have severe muscle symptoms or signs, nor elevation of CPK or anti-Jo-1 antibodies, their pulmonary involvement is refractory to conventional therapies including corticosteroid pulse therapy. The present patient also was resistant to cyclosporine as well as steroid therapy. Additional IVCY was effective and interstitial pneumonia gradually improved while prednisolone was tapered. 
Some reports have suggested that cyclosporine appears to be effective for interstitial pneumonia associated with PM/DM refractory to other immunosuppressive drugs when used early in the course of the disease $(2,5)$. In the present case, cyclosporine was effective for myositis. However, cyclosporine was not effective for interstitial pneumonia. It might be due to the long duration from onset of interstitial pmeumonia. In this patient, interstitial pneumonia had already occurred in 1993. It also might include various pathological findings. Therefore, her clinical findings might not be improved so promptly after administration of IVCY.

Cyclosporine and cyclophosphamide have different inhibitory mechanisms on immune system. Cyclosporine selectively affects lymphocytes and inhibits their cellular immunity (9). This immunosuppressant inhibits lymphocyte proliferation and cytokine production. Some reports showed that cyclosporine was effective for interstitial pneumonia of PM/DM in spite of lack of lymphocyte infiltration on BAL findings or histologicaly diffuse alveolar damage (DAD) cases. Cyclophosphamide acts as a cytotoxic drug for all dividing-stage cells including immune cells (10). Recently Schnabel et al (11) reported that IVCY improved interstitial lung disease irrespective of the response of BAL lymphocytes. The combination of these two agents, which have different immune suppressive actions (target cells, mechanism of action, etc.) seems to inhibit immune disorders more widely and more strongly.

The main histologic features of interstitial pneumonia associated with PM/DM were thought to be bronchiolitis obliterans organizing pneumonia (BOOP), usual interstitial pneumonia (UIP), nonspecific interstitial pneumonia (NSIP), and DAD $(12,13)$. With respect to this patient's pulmonary symptoms, BOOP was unlikely due to the lack of effectiveness of corticosteroid therapy and the presence of reticular shadows on CT scan. However, it was difficult to distinguish BOOP from other interstitial pneumonia clinically as numerous manifestation patterns have been reported recently in BOOP lesions, from a multiple spotty shadow pattern to a reticular shadow pattern. UIP could not be considered because honeycomb lung or marked fibrosis was not found on chest X-rays or CT scan. DAD was not suitable for this case because of the response to medications and the clinical course. Katzenstein and Fiorelli (13) proposed NSIP, the fourth interstitial pneumonia of unknown etiology, which is characterized by various proportions of interstitial inflammation and fibrosis that appear to occur over a single time span. The most presenting complient was dyspnea for several months and chest radiographs usually showed bilateral interstitial infiltrates. Thererfore, we proposed that this patient's findings were NSIP with clinical PM/DM. Yousem et al (14) recommended open lung biopsy for establishing the diagnosis of interstitial pneumonia. However, in the present case it was hazardous to perform an open lung biopsy due to her rapidly progressive respiratory distress. Since NSIP associated with PM/DM reveals a mixed pattern of various pathological features ranging from pure inflammation to fibrosis, it is necessary that immune disorders should be sup- pressed in NSIP with PM/DM (13). Several reports have suggested the effectiveness of immunosuppressive drugs such as azathioprine (4), cyclophosphamide $(3,4)$, or cyclosporine $(2$, 5) for steroid-resistant interstitial pneumonia associated with $\mathrm{PM} / \mathrm{DM}$ while contradictory results have also been published $(6,7)$. When the addition of one cytotoxic agent has failed, a combination of 2 agents (cyclosporine and azathioprine) has also been reported to be successful (15). It was suggested that more than two immunosuppressive agents, which play different inhibitory roles on the immune system, might have been necessary for the therapy of steroid-resistant interstitial pneumonia associated with PM/DM. We hope that various combinations of immunosuppressive agents will be applicable for the successful treatment of the steroid-resistant interstitial pneumonia associated with PM/DM.

\section{References}

1) Takizawa H, Ito K. Cyclophosphamide pulse therapy for rapidly progressive interstitial pneumonia in dermatomyositis. A new possibility for rescue? Internal Med 36: 448-449, 1997 (editorial; comment).

2) Maeda K, Kimura R, Komuta K, Igarashi T. Cyclosporine treatment for polymyositis/dermatomyositis: is it possible to rescue the deteriorating cases with interstitial pneumonitis? Scand J Rheumatol 26: 24-29, 1997.

3) Shinohara T, Hidaka T, Matsuki Y, et al. Rapidly progressive interstitial lung disease associated with dermatomyositis responding to intravenous cyclophosphamide pulse therapy. Intern Med 36: 519-523, 1997.

4) McCune WJ, Vallance DK, Lynch JP. Immunosuppressive drug therapy. Curr Opin Rheumatol 6: 262-272, 1994.

5) Gruhn WB, Diaz-Buxo JA. Cyclosporine treatment of steroid resistant interstitial pneumonitis associated with dermatomyositis/polymyositis. J Rheumatol 14: 1045-1047, 1987.

6) De Toro Santos FJ, Verea-Hernando H, Montero C, Blanco-Aparicio M, Torres Lanzas J, Pombo Felipe F. Chronic pneumomediastinym and subcutaneous emphysema: association with dermatomyositis. Respiration 62: 53-56, 1995.

7) Levy S, Hodgson HJF. Cyclosporine for dermatomyositis? [letter]. Ann Rheum Dis 48: 85-86, 1989.

8) Kohno N, Kyoizumi S, Awaya Y, Fukuhara H, Yamakido M, Akiyama M. New serum indicator of interstitial pneumonitis activity. Sialylated carbohydrate antigen KL-6. Chest 96: 68-73, 1989.

9) Amano K, Takano Y, Takeuchi T, et al. Cyclosporin A therapy for interstitial pneumonitis in patients with polymyositis/dermatomyositis (PM/DM). Jpn J Inflammation 17: 143-148, 1997.

10) Turk JL, Parker D, Cameron AE. The effect of cyclophosphamide on immunological control mechanisms. Int J Tissue React 6: 205-211, 1984.

11) Schnabel A, Reuter M, Gross WL. Intravenous pulse cyclophosphamide in the treatment of interstitial lung disease due to collagen vascular diseases. Arthritis Rheum 41: 1215-1220, 1998.

12) Tazelaar HD, Viggiano RW, Pickersgill J, Colby TV. Interstitial lung disease in polymyositis and dermatomyositis. Clinical features and prognosis as correlated with histologic findings. Amm Rev Respir Dis 141: $727-$ 733, 1990.

13) Katzenstein AL, Fiorelli RF. Nonspecific interstitial pneumonia/fibrosis. Histologic features and clinical significance. Amm J Surg Pathol 18: 136147, 1994.

14) Yousem SA, Colby TV, Carrington CB. Lung biopsy in rheumatoid arthritis. Amm Rev Respir Dis 131: 770-777, 1985.

15) Sauty A, Rochat T, Schoch OD, Hamacher J, Kurt AM, Nicod LP. Pulmonary fibrosis with predominant CD8 lymphocytic alveolitis and anti-Jo-1 antibodies. Eur Respir J 10: 2907-2912, 1997. 